\title{
Grado de Satisfacción de los adultos con los Programas Universitarios
}

\section{Satisfaction level of adults with University Programmes}

\author{
Ana FERNÁNDEZ-GARCÍA, José Luis GARCÍA LLAMAS y Gloria PÉREZ SERRANO \\ Universidad Nacional de Educación a Distancia
}

Recibido: Noviembre 2014

Evaluado: Marzo 2015

Aceptado: Marzo 2015

\begin{abstract}
Resumen
La presente investigación analiza el grado de satisfacción de una muestra de 874 alumnos/as de Programas Universitarios para Adultos-Mayores (PUM) de todo el Estado Español. La investigación se enmarca dentro de la psicología positiva, específicamente en la propuesta de Martin Seligman, creador de este movimiento, quien propugna que el principal objetivo de esta rama de la ciencia psicológica es investigar los aspectos saludables de la vida del ser humano. La metodología utilizada es mixta. El grado de satisfacción, recogido a través de la técnica de la encuesta, fue analizado mediante el programa estadístico SPSS 20.0 (análisis de segmentaciónCHAID) para la información cuantitativa; y el programa Atlas.Ti 6.0 (análisis textual y conceptual) para la información cualitativa. Los datos analizados revelan que el alumnado se encuentra altamente satisfecho con el Programa, el profesorado y sus compañeros/as. La discusión corrobora estos resultados, dado que también se han puesto de relieve en otros estudios.
\end{abstract}

Palabras clave: investigación educativa, formación continua, satisfacción, adulto, psicología positiva.

\begin{abstract}
This research analyses the degree of satisfaction of a sample of 874 students from University Programmes for adults and elderly, from Spain. The investigation is framed along the lines of positive psychology, specifically in Martin Seligman's proposal, founder of this movement, who advocates that the main objective of this branch of psychological science is to investigate the healthy aspects of human life. The methodology applied is mixed. The level of satisfaction, collected through the survey technique was analysed by the SPSS 20.0 (using method CHAID) statistical program for the quantitative data; and by the Programme Atlas.Ti 6.0 (textual and conceptual analyses) for the qualitative data. The analysed data reveal that students are highly satisfied with the programme, the teachers and their peers. The discussion confirms these results, since it has also stood out in others studies.
\end{abstract}

Keywords: educational research, continuous training, satisfaction, adult, positive psychology. 
El incremento constante de adultos mayores en la sociedad, en las últimas décadas, respecto a la población total, ha contribuido a que aumente la preocupación por la calidad de vida en relación a esta cohorte etaria.

Diversos autores, entre ellos, Martí, Martínez, Martí \& Marí (2007) advierten que "el aumento de las expectativas de vida de la población mayor, reflejo de las mejoras socioeconómicas y sanitarias registradas en el último tiempo, obliga a una preocupación por la calidad de vida de estas personas".

La disponibilidad de tiempo libre de esta población reclama, desde ámbitos sociales, la creación de propuestas y modalidades formativas que les aporten nuevas oportunidades, no como mero pasatiempo, sino que contribuyan al logro de su propio desarrollo personal y les permita seguir activos intelectualmente.

En este contexto surgen los Programas Universitarios para Adultos-Mayores (PUM). Su origen difiere en los distintos países y entornos en los que se ha implantado. Se conocen dos grandes tendencias: la francófona y la anglosajona. Ambas, aunque diferentes, tienen en común acercar la educación y la cultura a este colectivo. En España, los PUM nacen a partir de los años ochenta. En un corto espacio de tiempo han logrado establecerse en todo el territorio. Lirio \& Morales (2011) afirman que existen más de 50 programas y alrededor de 30.000 estudiantes que siguen estos estudios.

Como propósitos primordiales que se encomiendan a los podemos mencionar, entre otros, la actualización de conocimientos, enriquecimiento de su vida social, fomento del desarrollo de su creatividad...; todos ellos tienen como objetivo mejorar su calidad de vida y su nivel de satisfacción.

La investigación que presentamos se enmarca dentro de la Psicología Positiva, específicamente en la propuesta de Martin Seligman, creador de este movimiento, quien propugna que el principal objetivo de esta rama de la ciencia psicológica es investigar los aspectos saludables de la vida del ser humano.

A finales del s. XX, el profesor Seligman propone la creación de la Psicología Positiva como un nuevo campo de acción de la Psicología. Su misión es investigar los procesos que subyacen a las cualidades y emociones positivas del ser humano. Se amplía así el ámbito tradicional de actuación más centrado en el déficit. Estudia la normalidad e incluso las formas sobresalientes y superiores de la existencia. Esta nueva orientación psicológica se enmarca en el método científico propio de la Psicología y por tanto, sus hallazgos e investigaciones se validan con procedimientos rigurosos. En su obra de 2002, La auténtica felicidad, identifica los tres pilares de la felicidad: emoción positiva, vida comprometida y vida con significado (elementos con más posibilidad de medición que la felicidad). La verdadera felicidad deriva de la identificación y el cultivo de las fortalezas y virtudes (Peterson \& Seligman, 2004) que en el uso cotidiano se plasman en el trabajo, el amor, el ocio y la educación. Estos aspectos conviene tenerlos en cuenta en la formación de Adultos-Mayores para diseñar programas de intervención y técnicas dirigidas a desarrollar los valiosos recursos que las personas, los grupos y las comunidades, poseen. 
La Psicología Positiva es un movimiento singular que insiste en la construcción de competencias y en la prevención. Parece existir evidencia empírica para afirmar que determinadas características positivas como el optimismo, la satisfacción vital, las fortalezas y virtudes de las personas, las relaciones humanas, entre otras, actúan como barreras frente al déficit. En este sentido, se puede afirmar que investigaciones recientes han concluido que, aspectos como el nivel de ingresos económicos o el nivel de prestigio, influyen positivamente sobre la felicidad de los sujetos, pero sólo son relevantes hasta cubrir las necesidades básicas. Sin embargo, a partir de este nivel, los ingresos y el prestigio, parecen no aportar mayores niveles de felicidad. Este aspecto se recoge en la obra denominada Para el bien común de Daly \& Cobb (1989, que cita Álvarez 2001). Los autores mencionados definen el índice de Bienestar Económico Sostenible -mediante las correcciones en los gastos de consumo personal, teniendo en cuenta la variedad de factores ambientales y sociales-, a partir de la aplicación empírica al caso de EE.UU., y muestran la discrepancia existente entre la evolución del PIB y el incremento del bienestar.

Basándose en esta investigación, Álvarez (2001) defiende la hipótesis de la existencia de un umbral en la relación entre el crecimiento del producto total y el bienestar humano, de forma que superado aquel, un incremento del PIB no se traduce en un incremento del bienestar. Otros autores han obtenido resultados similares estudiando diferentes países desarrollados, tales como (Jackson \& Marks, 1996 citado en Álvarez, 2001).

En cuanto a las relaciones sociales, investigaciones como la de Fernández-García \& Ponce-De-León-Romero (2013) concluyen, que en lo que se refiere a las relaciones interpersonales, su cantidad y calidad aparece asociada a un mayor nivel de satisfacción percibido, dado que la investigación evidencia que las principales necesidades durante el envejecimiento son: salud, actividad física adaptada a las posibilidades de cada uno, independencia y participación en un grupo, asociación o club. Otros factores (Harker \& Keltner, 2001, citado en Tarragona, 2013), también aparecen relacionados con niveles de mayor o menor satisfacción, tales como la estabilidad emocional, el humor, la autoestima, etc.

Seligman (2011) propone cinco factores que contribuyen al bienestar y a la satisfacción personal. Los sintetiza con el acrónimo, en inglés, PERMA: Positivity (positividad), Engagement (involucramiento), Relationships (relaciones interpersonales), Meaning (significado), y Achievement (Metas Alcanzadas/Logro). A continuación, recogemos brevemente en qué consiste cada uno de estos elementos y de qué manera se relacionan con el grado de satisfacción del alumnado de los Programas Universitarios de Adultos-Mayores:

En primer lugar, la positividad hace referencia a experimentar emociones positivas; se relaciona con la tendencia a ver y juzgar los aspectos más favorables en orden al afrontamiento de la vida. Existe evidencia de que la positividad se relaciona con el bienestar físico y la longevidad (Tarragona, 2013). Otro aspecto, las relaciones interpersonales, son primordiales para el bienestar de las personas. Deben ser positivas $\mathrm{y}$, por ende, es necesario cuidarlas a cualquier edad para el logro de la plena integración social. La experiencia ha demostrado que, los Programas Universitarios, 
constituyen un espacio privilegiado para el encuentro y las relaciones interpersonales. Por su parte, el involucramiento alude a la conveniencia de que exista relación entre la capacidad y el nivel de dificultad en la realización de tareas. Si el nivel de reto es bajo, sentiremos aburrimiento; por el contrario, si la actividad es demasiado difícil, sentiremos frustración y ansiedad. Si somos capaces de realizar actividades asequibles, nos sentiremos satisfechos y nuestro estado emocional será óptimo. Aspecto esencial a tener en cuenta en los Programas Universitarios para Adultos-Mayores, que deben adaptarse a las características individuales de cada alumno. El cuarto aspecto es el significado, que se refiere al sentido que se dé a nuestra vida. Las personas que piensan que su vida tiene sentido, experimentan mayor bienestar y satisfacción. Además, ello influye en el control de sus vidas, rasgo relevante en los PUM. Las metas alcanzadas se relacionan con la satisfacción que sentimos cuando nos enfrentamos a retos y conseguimos logros. Alcanzar metas nos produce satisfacción, porque podemos desarrollar habilidades y sentirnos competentes. Los Adultos-Mayores, que no han tenido la oportunidad de acceder en su momento a los estudios superiores, esta experiencia les aporta una gran satisfacción personal.

Todo lo expuesto sirve de marco para contextualizar el intento por mejorar nuestro conocimiento sobre la formación de los Adultos-Mayores.

La presente investigación tiene como principal objetivo determinar el grado de satisfacción del alumnado de los Programas Universitarios de Adultos-Mayores. Así mismo, se han establecido como objetivos específicos los siguientes: 1) Analizar el perfil del alumnado que asiste a los PUM; 2) Comprobar el grado de satisfacción del alumnado con sus propios compañeros, el profesorado y los coordinadores y 3) Identificar el grado de satisfacción de los Adultos-Mayores con los Programas que se imparten en su centro universitario.

Los PUM han sido objeto de reflexión y estudio por parte de organismos, instituciones y profesionales. A continuación se presentan diversos estudios y análisis originados en torno a esta temática que contienen aspectos relacionados con el grado de satisfacción de los participantes:

Vecina Jiménez (2006) cita un importante estudio, realizado por Ostir, Markides, Black \& Goodwin, (2000), titulado: Emotional well-being predicts subsequent functional independence and survival. Evalúa el estado de salud y el estado emocional de 2.282 sujetos de más de 65 años, a los que se les hizo un seguimiento durante dos años. Los resultados mostraron que la experiencia de emociones positivas protegía a las personas mayores de los efectos más negativos del envejecimiento y de la incapacidad.

Puga, Rosero-Bixby, Glaser \& Castro (2007) realizaron una investigación denominada Red social del adulto mayor desde una perspectiva comparada: Costa Rica, España e Inglaterra. Analiza las redes sociales de apoyo del Adulto-Mayor en tres sociedades diferenciadas: latinoamericana, latino-europea y anglosajona. Se constata que las redes sociales operan sobre el bienestar a través del flujo del apoyo social. 
González (2009) ha investigado sobre, La evaluación como instrumento de mejora y calidad de los programas universitarios para mayores: ULPGC (Universidad de las Palmas de Gran Canaria). Uno de los objetivos primordiales de esta investigación consistió en analizar el grado de satisfacción de los estudiantes con el conjunto del programa: organización, gestión, funcionamiento, infraestructura, condiciones ambientales y accesibilidad. Otro objetivo se centró en identificar el grado de satisfacción con la parte académica del programa. Las conclusiones más relevantes muestran que los participantes manifiestan una alta satisfacción tanto con la dimensión académica como con el conjunto del programa, alcanzando un $99,4 \%$.

Benítez \& Domínguez (2010) estudiaron el bienestar subjetivo desde el proceso de longevidad satisfactoria, reto en la educación del adulto mayor. Su objetivo fue desarrollar un programa educativo con enfoque personológico, participativo, creativo y potenciador del desarrollo humano, en orden a propiciar el bienestar subjetivo y lograr una longevidad más satisfactoria. Este estudio se llevó a cabo en la cátedra universitaria de la Casa de los Abuelos (Cuba). El programa estaba constituido por diferentes talleres. Entre ellos, destacamos el denominado "El sentido de mi vida", cuyo objetivo fue valorar el nivel de satisfacción que en las esferas de la vida manifiesta el Adulto-Mayor, principal criterio para el envejecimiento exitoso.

Arnay, Marrero \& Fernández (2011) han realizado un trabajo de investigación denominado ¿Por qué está satisfecho el alumnado de la Universidad para Mayores?: análisis comparativo con el alumnado de la Licenciatura de Pedagogía. Entre los criterios que analizaron se encuentran los procesos de enseñanza-aprendizaje, la valoración subjetiva del aprendizaje y la autopercepción de habilidades y destrezas personales relacionadas con el mismo. El alumnado manifiesta un alto grado de satisfacción con la experiencia educativa. Por otro lado, los resultados han mostrado que el grado de satisfacción general del alumnado mayor es superior al del alumnado de la licenciatura.

Morales (2011) analizó la figura El profesor tutor de la UNED Senior. Este estudio se desarrolla en el contexto de la Universidad para Adultos-Mayores en todo el Estado español. Realiza una investigación de corte evaluativo, con el fin de identificar aspectos como: el perfil docente, la adecuación de su metodología didáctica, estilo de enseñanza y el grado de satisfacción de los implicados. Los resultados obtenidos ponen de manifiesto que el alumnado se encuentra muy satisfecho con el Programa, obteniendo una media de 3,41 en una recorrido de 1 a 4.

Por su parte, García (2011) ha realizado un trabajo de investigación denominado Variables psicosociales que inciden en la calidad de vida del alumnado participante en PUM. Esta investigación aporta nuevos datos al estudio de la vejez al profundizar en el conocimiento de la calidad de vida de los mayores en situaciones de aprendizaje. El objetivo de este estudio es conocer la calidad de vida y las variables psicosociales relacionadas con el alumnado mayor que asiste a estos Programas. Como resultado se confirma que este alumnado posee una calidad de vida con indicadores positivos en referencia a un adecuado apoyo social, bienestar psicológico, autoestima personal y colectiva, optimización del ocio y tiempo libre, y la asunción de un nuevo rol generacional junto con nuevas competencias psicosociales. 
Sitges \& Bonete (2011) estudiaron el Estado emocional y la satisfacción de un programa universitario para mayores de la Universidad Miguel Hernández de Elche. La finalidad de esta investigación consiste en estudiar la incidencia de los PUM sobre el estado de ánimo de los sujetos que participan en ellos. Los resultados muestran altas puntuaciones sobre la satisfacción por parte de los sujetos al cubrir las necesidades y expectativas de formación. Datos que inciden de forma positiva en la mejora de la calidad de vida.

Cuenca Infante, (2011) analizó, La satisfacción del adulto mayor en correspondencia con su régimen de vida. Realiza un estudio en los consejos populares del municipio de Holguín (Cuba), sobre la satisfacción del Adulto-Mayor en correspondencia con su régimen de vida. Su objetivo es evaluar si las actividades que realizan satisfacen sus expectativas y contribuyen a elevar su calidad de vida. Las conclusiones delatan que los mayores participan poco en los círculos sociales, lo que incide en detrimento de su calidad de vida y por ende de su salud.

Cuenca París (2013) realizó una investigación, cuyo objetivo consistió en evaluar un Programa Universitario para personas mayores, en todo el Estado Español. La investigación identifica variables tan relevantes como: las relaciones interpersonales, las habilidades sociales, el clima, los cauces de participación, los intereses, expectativas y satisfacción de los mayores... Investigación que permitió conocer y estudiar la realidad, desde la perspectiva del grado de satisfacción de los participantes, para mejorarla y transformarla.

Padilla-Góngora, Santiuste \& Ruiz (2013) han investigado sobre Empowering Marginalized elders: Proyecto Grundtvig, llevado a cabo en cinco países europeos (Chipre, Hungría, Alemania, Rumanía y España). Su finalidad es describir las transformaciones que se están produciendo en la situación de las personas mayores frente a la exclusión social. Entre las acciones encaminadas a fortalecer las carencias de las personas mayores destaca la educación para Adultos. Demuestran que el aprendizaje para toda la vida es una de las oportunidades más efectivas para la inclusión social. Permite a los ancianos marginados la oportunidad de aprender, ser más autosuficientes, mejorar el bienestar psicosocial y su satisfacción vital.

En la temática de las investigaciones estudiadas se ubica también la que presentamos a continuación.

\section{Instrumento de la investigación}

El cuestionario, instrumento de recogida de datos, sirve en este estudio de nexo de unión entre los objetivos de la investigación y la realidad de la población encuestada. Se dirige a personas individuales, pero lo que nos interesa, en esta ocasión, son los datos agrupados, es decir, el estudio conjunto de los datos recogidos sobre muchos individuos para establecer clases o tipos, obteniendo de este modo el perfil de la población analizada.

Para llevar a cabo su construcción hemos contemplado dos tipos de preguntas: las de respuesta cerrada (42 ítems escala tipo Lickert) y abierta (2 ítems). Las primeras, 
fueron codificadas previamente, con valores numéricos, con el objetivo de obtener respuestas precisas. Las dimensiones del cuestionario se refieren a aspectos vinculados a la motivación para el aprendizaje, grado de satisfacción con el profesorado, el programa, el coordinador y con sus propios compañeros/as. Las preguntas abiertas ofrecen la oportunidad de profundizar en las opiniones y valoraciones de los sujetos, en los puntos que se hace visible la opinión personal. Éstas recogen información sobre las fortalezas y debilidades del programa. La respuesta a estas preguntas nos ha permitido realizar el análisis de carácter cualitativo. El cuestionario fue aplicado por encuestadores preparados adecuadamente.

La validez del cuestionario se ha determinado a través de la validez de contenido. Ha sido revisado por veinte coordinadores de órganos directivos de los PUM de cada Comunidad Autónoma y once expertos en metodología de investigación. Mediante este procedimiento se ha constatado que los ítems recogidos son fiel reflejo de las conductas o fenómenos de la población objeto de estudio y, en consecuencia, son coherentes con los objetivos del trabajo empírico.

La fiabilidad, se expresa en función de un coeficiente de correlación. Para proceder al cálculo numérico hemos recurrido al empleo del ordenador mediante el paquete estadístico SPSS 20.0. Concretamente se ha utilizado el subprograma reliability analysis-scale (Alpha). Este procedimiento contempla el número de elementos del cuestionario, además de las varianzas de los elementos y la total. Así pues, la fiabilidad del cuestionario fue obtenida mediante consistencia interna a través del coeficiente Alpha de Cronbach que obtuvo un valor de 0,859. Este dato confirma la alta consistencia interna de sus elementos.

\section{Participantes}

La población, universo de esta investigación, está constituida por todo el alumnado participante en los PUM, curso 2013-14. La muestra, seleccionada mediante un muestreo probabilístico está constituida por 874 alumnos, mayores de 55 años, que pertenecen a las siguientes Comunidades Autónomas repartidas por todo el territorio español (Madrid, Cataluña, Galicia, Aragón, Valencia, Asturias, Islas Baleares, Canarias, Cantabria, Navarra, Andalucía y Extremadura) y un centro europeo (BernaSuiza).

\section{Procedimiento}

La investigación adoptó un proceso en dos fases. La primera, supuso el contacto con los coordinadores y el profesorado de los respectivos PUM con el objetivo de que conocieran la investigación y, de este modo, obtener su aprobación. La segunda fase, consistió en la aplicación de los cuestionarios por encuestadores preparados y gracias a la colaboración del profesorado y los coordinadores. La recolección de los datos se llevó a cabo durante los meses de enero a marzo de 2014. El alumnado aceptó participar voluntariamente tras garantizárseles la confidencialidad de la información recogida y que sus opiniones solamente se utilizarían a efectos de investigación. 


\section{Método}

El método, como expresa Pérez Serrano (2011), es un elemento esencial en la estructura del trabajo científico. Es imprescindible para la búsqueda de un resultado que no está determinado. En nuestro estudio, teniendo en cuenta los objetivos, se consideró pertinente recurrir a la complementariedad metodológica, pues nos ofrecía una visión más completa de la realidad analizada, ello nos ha permitido utilizar tanto la metodología de carácter cuantitativo como cualitativo. Estas metodologías han servido para describir, comprender, analizar y explicar las variables que inciden en el grado de satisfacción del alumnado de los PUM.

\section{Análisis estadísticos}

El análisis cuantitativo de los datos se ha llevado a cabo siguiendo las etapas que se describen a continuación:

Por medio de los análisis de tipo descriptivo se establecen los perfiles de los participantes en cuanto a sus rasgos personales (sexo, edad, nivel de estudios, formación inicial, etc.). Así mismo, nos permiten establecer diversas valoraciones en torno a los diferentes elementos del programa. Aporta datos sobre frecuencias absolutas, relativas y acumuladas y sus valores medios, apoyándolos con representaciones gráficas.

A través del análisis multivariante podemos depurar aún más el cuestionario de recogida de datos. Mediante el análisis de segmentación se crea un modelo de clasificación basado en árboles (árbol de decisiones). Este procedimiento pronostica valores de una variable dependiente (criterio) basada en valores de variables independientes (predictores). De esta manera, nos proporciona herramientas de validación para los análisis de clasificación exploratorios y confirmatorios. En esta investigación se ha utilizado el método de crecimiento CHAID (CHi-square Automatic Interaction Detection). En cada paso, CHAID, presentará la interacción más fuerte de la variable independiente con la variable dependiente, fundiéndose las que no sean significativas. Este método examina todas las divisiones posibles y muestra la salida mediante un "árbol" clasificatorio de fácil interpretación. Para realizar este análisis se ha utilizado el programa estadístico SPSS 20.0. Este programa detecta automáticamente las interacciones mediante la prueba de chi-cuadrado. Así mismo, los valores de significación se corrigen mediante el método de Bonferroni.

El análisis cualitativo de la información se ha apoyado en el software Atlas. Ti 6.0. Este programa es una herramienta informática que facilita el trabajo de ordenar y organizar la información recogida para alcanzar una visión de conjunto del objeto de estudio.

Siguiendo a Pérez \& Pérez (2010), la investigación cualitativa es una vía que intenta acercarse al conocimiento de la realidad a través de la observación participante en los hechos o a través de los discursos. Los métodos cualitativos intentan tomar conciencia de la realidad, observarla en su contexto natural y comprenderla en todas sus dimensiones, más que explicarla y predecirla. 
Una vez recogidos los datos, se procedió a sistematizar la información. El trabajo se organizó en tres etapas, a saber: la primera de ellas consistió en la categorización de la información recogida mediante las preguntas abiertas del cuestionario; a continuación se crearon redes de relaciones entre las categorías denominados diagramas de flujo y/o mapas conceptuales. Finalmente se procedió a su teorización: las relaciones entre las categorías vienen respaldadas por el uso de operadores booleanos, semánticos y de proximidad.

\section{Resultados}

En primer lugar se describe la población a la que pertenece el alumnado de los diferentes PUM, con relación a su situación actual, sexo, edad, nivel de estudios y situación laboral, respectivamente.

La situación actual del alumnado revela que el $61,1 \%$ son casados; un $15,2 \%$ son viudos; el $14,4 \%$ solteros y el 5,3\% no contesta. En lo que se refiere al sexo, la mayoría son mujeres el $63,5 \%$, en tanto que el $32,0 \%$ son varones y el $4,5 \%$ no contesta.

En cuanto a los grupos de edad, el porcentaje más elevado se encuentra en la franja entre 61-65 años, que alcanza el 30\%, seguido de la franja 66-70 con un 27,1\% y los de menos de 60 años logran un porcentaje del $23 \%$. A partir de los 71 años los porcentajes van menguando y, podemos observar, cómo sólo el 7,6\% corresponde a los mayores de 76 años. Por tanto, la década de 60 a 70 es la franja de edad en la que se constata una mayor participación.

Referente al nivel de estudios, el $41,5 \%$ manifiesta que posee estudios universitarios, seguido del $32,8 \%$ con estudios secundarios, finalmente el $22,1 \%$ posee estudios primarios, en tanto que el 3,4\% no contesta. La situación laboral en la que se encuentran en la actualidad los Adultos-Mayores, también es muy diversa, aunque predominan los jubilados en un $71,69 \%$, si bien un $12 \%$ todavía están trabajando, un $9,6 \%$ se encuentran en paro y un $6,8 \%$ no responde.

A continuación se describe el "grado de satisfacción" del alumnado con el profesorado, el coordinador, los compañeros y su propio grado de satisfacción con los PUM. Los resultados obtenidos revelan el alto grado de satisfacción con el profesorado, media de 8,86; con los compañeros, media 8,50; con los Programas Universitarios, media 8,59. Algo más baja es la media alcanzada con el coordinador de 6,79 . Este último dato pone de relieve que los alumnos tienen poco contacto con el coordinador y desconocen sus funciones. Los valores se visualizan en los gráficos siguientes: 


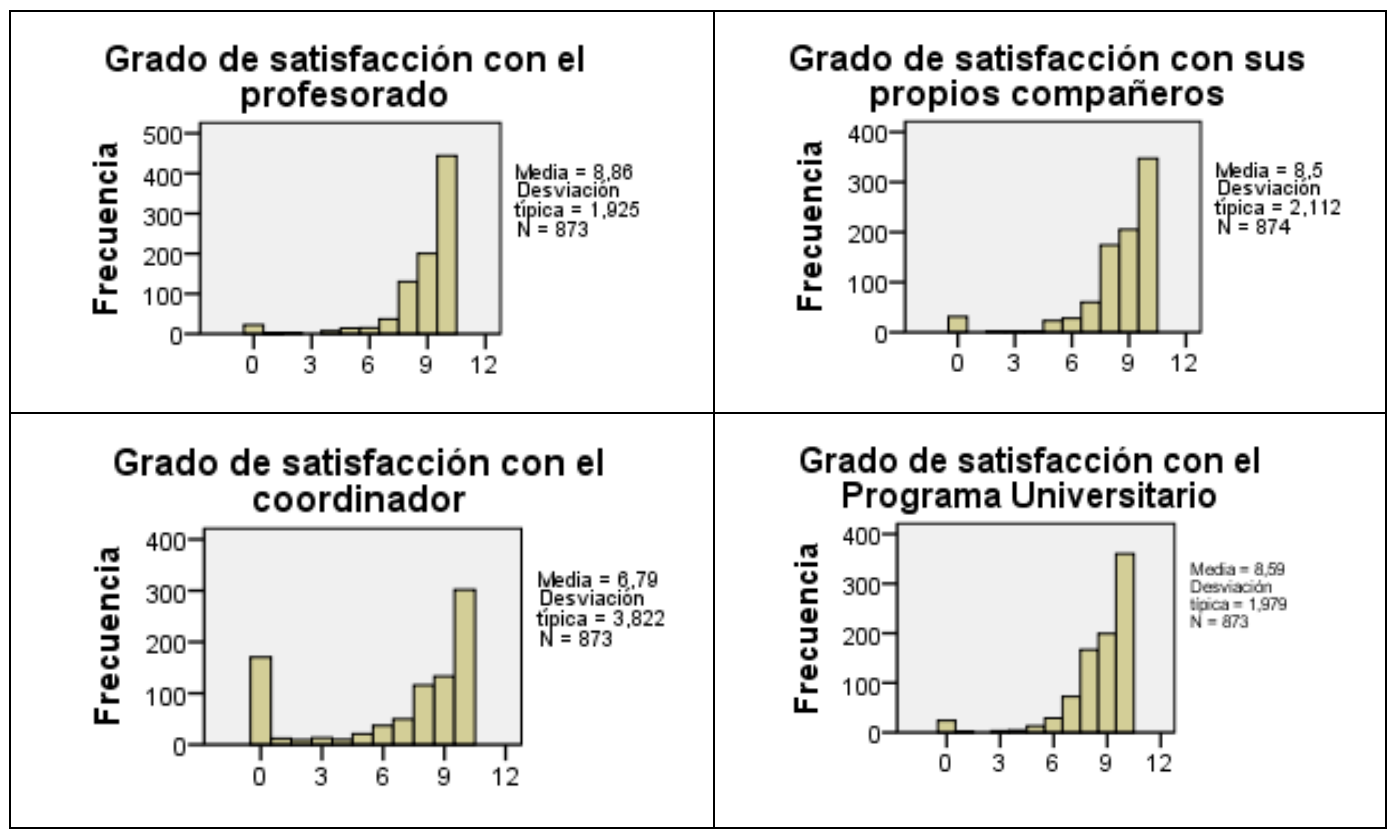

Tabla 1. Gráficos de medias (grado de satisfacción).

Fuente: Elaboración propia.

Así mismo, se ha realizado un análisis de contingencia en el que se han cruzado las variables "grado de satisfacción del alumnado con el profesorado" por Comunidades Autónomas. Conviene tener presente que en la variable grado de satisfacción se ha agrupado "nada" y "poco" (hasta 5 y menos); bastante (6 y 7) y mucho (8, 9 y 10). En general, los Alumnos de las diferentes Comunidades, repartidos por toda la geografía española y en el extranjero, están muy satisfechos con el profesorado. Los Alumnos que están plenamente satisfechos (100\%) pertenecen a las siguientes comunidades: Cataluña, Extremadura y Madrid.

A continuación se indica, de mayor a menor, el grado de satisfacción del alumnado de otras Comunidades Autónomas: Canarias (96,2\%), Comunidad Valenciana (95,5\%), Galicia (95,1\%), Asturias (92,3\%), Navarra (92,1\%), Aragón (88,5\%).

Un elemento importante en educación a cualquier edad y, también, en los AdultosMayores, es la "relación con los compañeros". En este sentido nos pareció de gran interés averiguar el grado de satisfacción del Alumnado con sus compañeros teniendo en cuenta la variable Comunidad Autónoma. Los resultados obtenidos revelan lo que se indica a continuación.

Los Alumnos de los Programas Universitarios se encuentran satisfechos de compartir esta experiencia educativa con sus compañeros. Cabe destacar que los Alumnos de las CA de Galicia (99\%), Madrid (95,5\%), Valencia (95,5\%) y Cataluña $(90,5 \%)$, se encuentran muy satisfechos con sus compañeros. Por su parte, se hallan 
muy satisfechos con los coordinadores de los centros ubicados en las comunidades de Galicia (95,1\%), Aragón (92,3\%) y Madrid (90,9\%). Conviene subrayar que el AdultoMayor tiene poca relación con el coordinador por lo que le resulta difícil valorarlo. En algunas ocasiones, tan solo se han encontrado con él, en la inauguración del curso y quizás en la entrega final de los certificados.

Con las respuestas a la pregunta relativa al grado de satisfacción se ha realizado el análisis de segmentación que aporta datos de especial interés para la investigación. Mediante este análisis se creó un modelo de clasificación basado en árboles (árbol de decisiones).

En primer lugar (Gráfico 1), se ha solicitado al Alumnado que exprese su "grado de satisfacción con el profesorado" (variable dependiente). En este sentido aparecen tres aspectos (variables independientes). El primero responde a "lo que esperaba". Le sigue, "el profesorado satisface mis expectativas". Por último, al alumnado le gusta que "el profesor demande su participación". Por ello, manifiestan el agrado que les proporciona que el profesor pida su participación en clase. Finalmente valoran mucho que sus Programas Universitarios, les hayan servido para aprender más.

También se le preguntó sobre su grado de satisfacción con sus propios compañeros. En este sentido el árbol de segmentación presenta los aspectos que se muestran más relevantes para definir esta categoría. Entre ellos destaca el Programa Universitario "me ha servido para conocer a otras personas" y "lo recomendaría a mis amigos". Se trata de expresiones que reflejan su grado de satisfacción.

En cuanto a la valoración que realizan los Alumnos sobre el Centro y el coordinador, conviene subrayar que se hallan altamente satisfechos los Alumnos que pertenecen a las comunidades de: Cataluña, Aragón, Comunidad Valenciana, etc. Se destaca como mejor valorado el Centro de A Coruña de la CA de Galicia. Convendría reflexionar en el resto de los centros los aspectos a mejorar para incrementar el grado de satisfacción del Alumnado. Los centros mejor valorados son aquellos en los que el coordinador presta atención al fomento de las relaciones interpersonales del Alumno, propicia las actividades y salidas culturales y la ocupación creativa y enriquecedora del tiempo libre.

El Alumnado muestra su satisfacción con el PUM en la medida en la que le hace sentirse útil y además responde a lo que esperaba. Así mismo, recomendarían estos Programas a otras personas. 


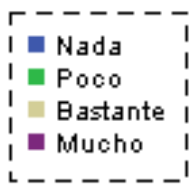

$$
\begin{aligned}
& \text { Grado de satisfacción con el } \\
& \text { profesorado (agrupado) }
\end{aligned}
$$

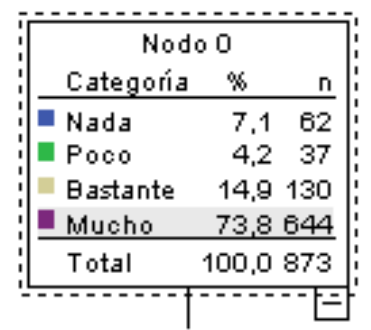

Responde a lo que yo esperaba

Valor $P$ corregido $=0,000$, Chicuadrado $=225,686, \mathrm{df}=2$
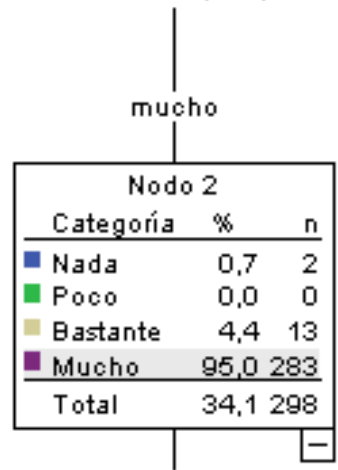

El profesor pide participación Valor $\mathrm{P}$ corregido $=0,002$, Chicuadrado=12,796, $d t=1$

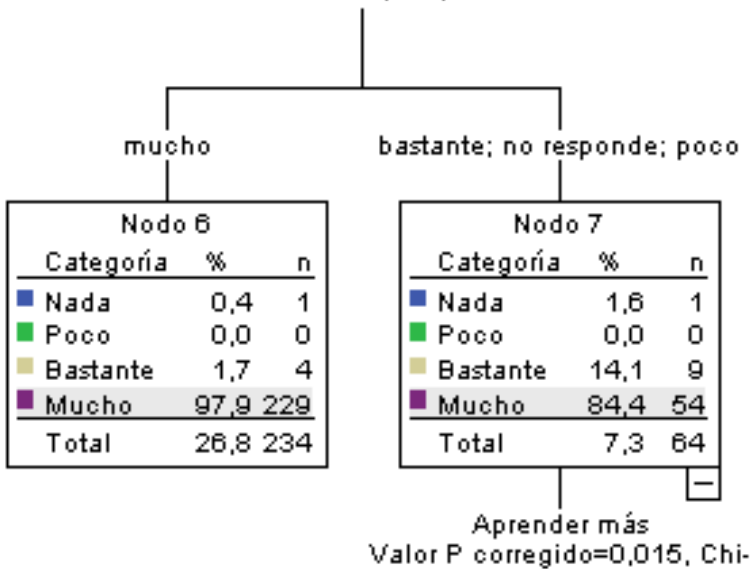

Gráfico 1. Árbol de Segmentación (Grado de satisfacción profesorado).

Fuente: Elaboración propia. 


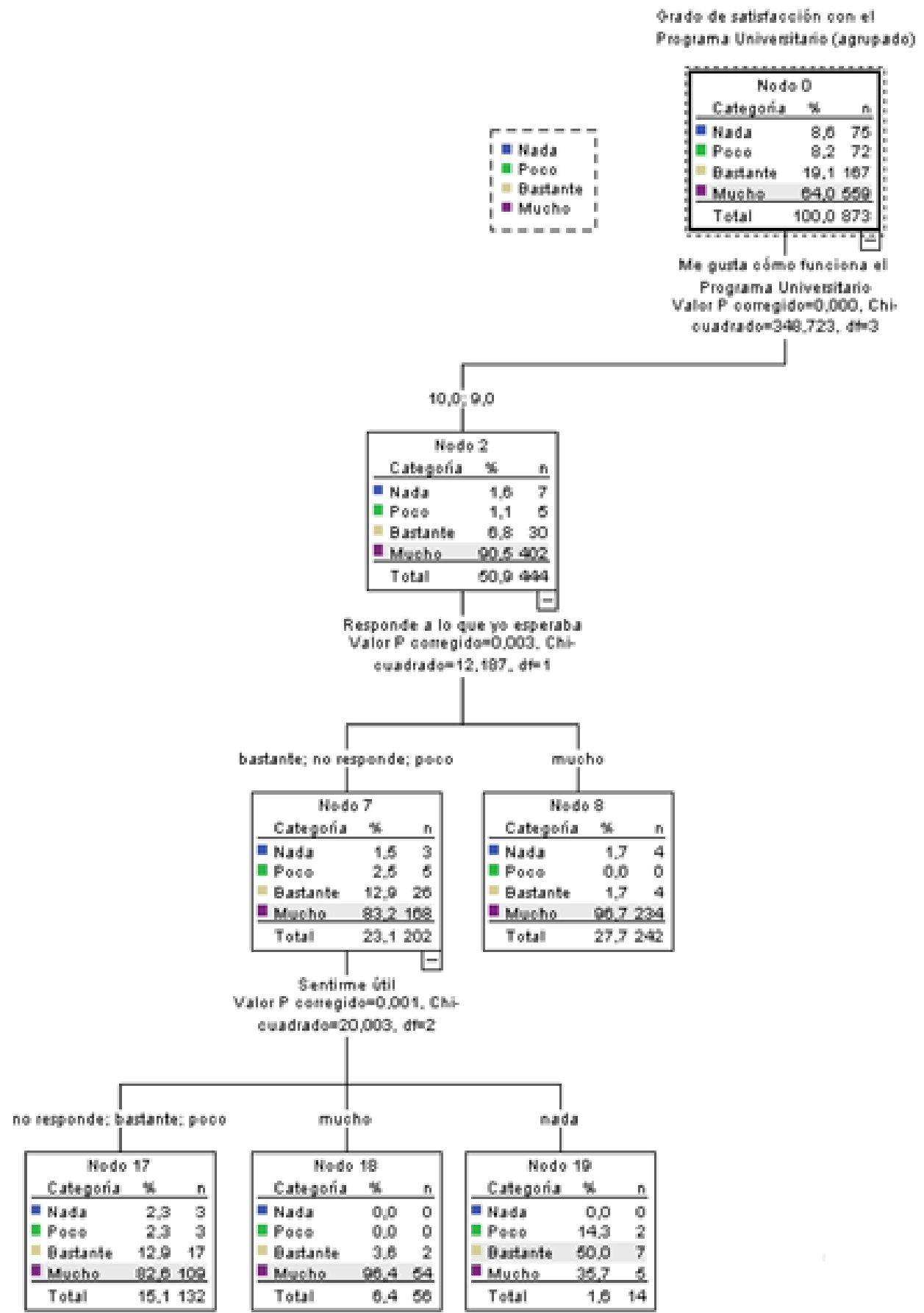

Gráfico 2. Árbol de Segmentación (Grado de satisfacción programas universitarios). Fuente: Elaboración propia. 
A continuación, se presenta la información cualitativa, por medio de las opiniones del alumnado de los PUM expresadas a través de las preguntas abiertas del cuestionario. Estas preguntas hacen referencia a los aspectos que más le satisfacen de sus respectivos Centros, así como a los que, según su opinión, necesitarían mejorar. Los resultados a ambas preguntas se muestran mediante matrices que recogen las categorías obtenidas una vez realizado el tratamiento de datos cualitativo con el programa Atlas. Ti 6.0.

Entre los aspectos que más le satisfacen de sus respectivos centros, son: en relación a la misión y finalidad del programa, afirman que "refuerza la autoestima", "la alegría de vivir y aprender", así como, la "facilidad de poder asistir" todo el que esté interesado. En cuanto a la organización y metodología, el alumnado destaca la "calidad humana y profesional" del profesorado, "las explicaciones", "los contenidos" y "las actividades". Como materias, cobra relevancia "la música y las humanidades", entre otras. Respecto al ambiente y las relaciones humanas destacan el "buen ambiente entre el alumnado, profesorado y personal de servicios", "el compañerismo" y "la familiaridad".

Hemos seleccionado algunas citas textuales que muestran lo que más les satisface a los estudiantes: "Refuerza la autoestima, el sentirme mejor, más activo intelectualmente y más satisfecho, además de aprender". "La independencia personal que se logra". "Ayuda mucho a la vida cotidiana". "El buen ambiente reinante entre alumnos y profesores". "La cordialidad, el compañerismo, la convivencia, el ambiente de clase", "el conocer a otras personas con intereses similares a los míos". El "trato humanitario del profesorado". Su "atención y amabilidad". Su "sencillez y dinamismo". "La coordinación entre el profesorado, personal de servicios y los alumnos".

En cuanto a los aspectos a mejorar en los PUM mencionan los organizativos. En general desearían cambios en: "mejorar la dotación de recursos y lograr un funcionamiento más adecuado"; solicitan que se "organicen cursos de verano", "distribuir y agrupar a los alumnos según conocimientos e intereses". En cuanto al programa y la metodología, afirman que "les gustaría realizar más actividades extraescolares y que éstas sean amenas", "más actividades culturales", "proponer y realizar talleres prácticos". En relación a la temporalización existen dos opiniones contrapuestas; por un lado, las que solicitan más horas de clase. Por otro lado, los que desearían que el tiempo de clase se redujese al considerarlo excesivo. En cuanto a las cuestiones administrativas solicitan que la información sea más clara y se dé más publicidad, información y apoyo. Finalmente, por lo que se refiere a las instalaciones, les gustaría, en algunos casos mejorar la accesibilidad.

\section{Discusión}

La mujer suele tener una mayor presencia que el varón en los PUM. Esto viene corroborado por diversas investigaciones realizadas, entre ellas las de Cuenca Paris (2013) y González (2009) quienes concluyen que existe una elevada proporción de mujeres que se matricula en estos Programas. Así mismo Vilaplana (2010) realizó un 
estudio en todo el territorio español, afirmando que los hombres manifiestan una menor predisposición a participar en programas de formación. De estos datos se deduce que las mujeres parecen más interesadas en seguir formándose, quizás, porque han tenido menos oportunidades. Se constata una cierta feminización en los PUM. De ello se puede concluir que la mujer necesite conquistar cotas más altas de formación que los varones.

En general, el grado de satisfacción del alumnado respecto del profesorado es muy elevado en todas las investigaciones consultadas. Este dato se ratifica también en nuestra investigación puesto que ha alcanzado una media de 8,86 en una escala de 1 a 10. Aspecto que se corrobora en las investigaciones realizadas por Sitges \& Bonete (2011), al indicar que los alumnos se encuentran muy satisfechos con la docencia recibida, obteniendo una media de 4,14 sobre 5 en una escala de respuesta tipo Lickert. Las investigaciones de Morales (2011) y Cuenca Paris (2013) también revelan que el grado de satisfacción del Adulto-Mayor con el Profesorado es muy elevado. Hallazgo también encontrado en la investigación de González (2009), que indagó sobre la valoración global que otorga el estudiante de Programas de Mayores al profesor, obteniendo un grado de satisfacción del 67,3\% del total del alumnado. El grado de satisfacción de los Adultos-Mayores con el profesorado es muy superior al obtenido en otros niveles educativos.

Otra de las dimensiones investigadas, hace referencia a la participación del alumnado. En este sentido Mouriño \& Benítez (2011) declaran, que se ha comprobado que la calidad de vida se encuentra estrechamente relacionada con la participación. En las investigaciones ya mencionadas, la participación de los adultos-mayores en los programas de formación les ha proporcionado satisfacción al poner en juego sus capacidades y desarrollar su personalidad. Estos adultos conceden mucha importancia a llegar a conseguir una mayor autonomía, mejor control personal y la elección de un estilo de vida. Fernández-García \& Ponce-De-León-Romero (2013) afirman que la pertenencia a un grupo, asociación o club, evita el aislamiento y mejora la capacidad de relación, incidiendo directamente en la percepción de la satisfacción y bienestar de la persona, como vínculo importante para compartir sus temores, alegrías o preocupaciones. Por ello, es muy importante asegurar la implicación activa de los Adultos-Mayores en la vida social y en el entorno donde se desenvuelven. En esta misma línea, De-Juanas, Limón \& Navarro (2013) han destacado que, “...la importancia del ejercicio físico, de las relaciones sociales, del ocio y de la formación, entre otros, en esta etapa de la vida", como elementos que potencian el bienestar psicológico y la calidad de vida en este sector de población. Londoño, Hernández, Alejo \& Pulido (2013) evidenciaron la relación entre el optimismo y las expectativas hacia el futuro, específicamente en el aumento de la sensación de control y de dar sentido a las experiencias vividas.

La investigación realizada nos permite afirmar que las relaciones con los compañeros proporcionan un alto grado de satisfacción y de bienestar personal. Se ha demostrado que es un elemento importante en educación a cualquier edad. El alumnado de los PUM se muestra satisfecho al tener la oportunidad de conocer a otras personas, aspecto que corroboran también otras investigaciones como las realizadas 
por Morales (2011) y Cuenca Paris (2013). Es importante destacar que gracias a los hallazgos encontrados en las dos últimas décadas, en la actualidad, el efecto beneficioso de las relaciones sociales sobre la salud está ampliamente reconocido en la gerontología y la salud pública (Puga et al., 2007). En este aspecto inciden otras investigaciones (Vecina Jiménez, 2006) al señalar que las emociones positivas se relacionan con la salud de manera satisfactoria.

Es significativo incentivar, así mismo, las relaciones intergeneracionales que se establecen en los diferentes centros universitarios (Pérez Serrano, 2013). Los AdultosMayores no solo toman contacto con personas de su misma edad, sino con un conjunto más amplio de individuos de diferentes edades, por el hecho de compartir el mismo entorno universitario. Ello da lugar a un enriquecimiento mutuo, donde el mayor tiene la oportunidad de compartir su experiencia y sabiduría con los más jóvenes, aspecto gratificante que refuerza la autoestima y el optimismo del Adulto-Mayor al sentirse copartícipe de la comunidad universitaria y por ende de la sociedad.

De modo global se puede indicar que los Adultos-Mayores se hallan muy satisfechos con sus respectivos Programas Universitarios, tal y como queda de manifiesto en investigaciones tales como, González (2009); Morales (2011); Arnay et al. (2011); Sitges \& Bonete (2011); Cuenca Paris (2013). Así mismo, el Alumnado muestra su satisfacción con el Programa, en la medida en la que le hace sentirse útil y responde a lo que esperaba. Es tal el grado de satisfacción que "lo recomendarían a otras personas". Se ratifica en investigaciones como las de González (2009); Morales (2011) y Cuenca Paris (2013).

Por lo que respecta a la organización y la metodología didáctica, el alumnado destaca que le satisface las explicaciones aportadas por el profesorado, así como también los contenidos seleccionados. Se llevan a cabo metodologías activas y participativas, basadas en la experiencia (continuación de aprendizajes adquiridos). Un aspecto a modificar, sentido por el alumnado, sería una mejor distribución y agrupamiento de los alumnos, teniendo en cuenta el nivel de conocimientos y sus intereses. Ello les permitiría un aprovechamiento óptimo del tiempo de clase, al estudiar contenidos adecuados a su nivel formativo. Las materias más satisfactorias para el alumnado son, la música y las humanidades.

También se le preguntó al alumnado sobre los aspectos que convendría cambiar para lograr un mejor funcionamiento de los PUM. Los Adultos-Mayores demandan la realización de más actividades culturales y que éstas sean más amenas, tales como visitar el patrimonio artístico, realizar cursos de verano, talleres prácticos, entornos ambientales, cine, teatro y otros. Investigaciones realizadas sobre el tema, Cuenca Paris (2013), Morales (2011), Benítez \& Domínguez (2010), ponen de relieve el gran interés de los Adultos-Mayores en estas actividades.

En cuanto a los aspectos organizativos, el alumnado investigado solicita cambios en la mejora de la dotación de recursos y su adecuado funcionamiento. Aspecto que coincide con otras investigaciones sobre Programas de Adultos-Mayores tales como, Morales (2011), donde los usuarios reclaman la posibilidad de contar con una mayor disponibilidad de recursos, así como mejoras en las instalaciones (mobiliario, etc.); y 
Cuenca Paris (2013) al señalar que los Alumnos-Mayores perciben que ciertos aspectos de los Centros y de las aulas no son adecuados a su edad, por sus limitaciones físicas y sensoriales. No obstante, otras investigaciones muestran resultados divergentes, como recoge González (2009) al señalar que las instalaciones de los Programas Universitarios estudiados, han sido adaptadas a sus necesidades llevando a cabo planes de reforma de edificios.

Como conclusión final, estamos en condiciones de afirmar que esta investigación ha conseguido el objetivo que pretendía, demostrar que los adultos mayores experimentan una gran satisfacción al participar en los Programas Universitarios diseñados para este grupo de población. Resaltan las posibilidades que le ofrece dicho programa para su realización personal y social, poniendo énfasis en la fuerza y significación de los vínculos e interacciones sociales como palanca de bienestar social. La participación en los Programas de formación les ha servido para aprender más, abrirse a nuevos horizontes, conocer a gente nueva, envueltos en un clima de compañerismo y familiaridad. Esta experiencia les hace sentirse útiles y reforzar su autoestima. Es necesario sumar esfuerzos para seguir investigando en la mejora de estos Programas, así como incentivar y fomentar metodologías creativas que ofrezcan nuevas vías de innovación. Todo ello redundará en una mayor calidad de vida de los adultos mayores y de toda la sociedad.

\section{Referencias bibliográficas}

ÁlVAREZ CANTALAPRIEDRA, S. (2001). El debate de las necesidades en la era de la globalización: un análisis del patrón de consumo alimentario español en los últimos veinticinco años. Madrid: Universidad Complutense de Madrid.

ARNAY PUERTA, J., MARRERO ACOSTA, J. \& FERNÁNDEZ ESTEBAN, I. (2011). ¿Por qué está satisfecho el alumnado de la Universidad para Mayores?: un análisis comparativo con el alumnado de la Licenciatura de Pedagogía. En Aprendizaje a lo largo de la vida, envejecimiento activo y cooperación internacional en los programas universitarios para mayores: IV Congreso Iberoamericano de Universidades para Mayores, CIUUM 2011: Alicante del 27 al 30 de junio de 2011. ISBN 978-84-615-1408-3. Vol. I, 175-189.

BENÍTEZ RÍOS, M. C. \& DOMÍNGUEZ ORTEGA, A. (2010). El bienestar subjetivo desde le proceso de longevidad satisfactoria: un reto en la educación del adulto mayor. En Contribuciones a las Ciencias Sociales. Recuperado el 21 de noviembre de 2013, de http://www.eumed.net/rev/cccss/09/brdo.htm

CUENCA INFANTE, D. M. (2011). La satisfacción del adulto mayor en correspondencia con su régimen de vida. En Aprendizaje a lo largo de la vida, envejecimiento activo y cooperación internacional en los programas universitarios para mayores: IV Congreso Iberoamericano de Universidades para Mayores, CIUUM 2011: Alicante del 27 al 30 de junio de 2011. ISBN 978-84-615-1408-3. Vol. II, 799-807. 
CUENCA PARIS, E. (2013). Evaluación del Programa Universitario para Mayores UNED-Senior. Madrid: UNED.

DE-JUANAS OLIVA, A., LIMÓN MENDIZÁBAL, M. R. \& NAVARRO ASENCIO, E. (2013). Análisis del bienestar psicológico, estado de salud percibido y calidad de vida en personas adultas mayores. Pedagogía Social. Revista Interuniversitaria, 22, 153-168.

FERNÁNDEZ-GARCÍA， T. \& PONCE-DE-LEÓN-ROMERO, L. (2013). Envejecimiento Activo: Recomendaciones para la intervención social desde el modelo de gestión de casos. Portularia, 13 (1), 87-97.

GARCÍA GONZÁLEZ, A. J. (2011). Variables psicosociales que inciden en la calidad de vida del alumnado participante en programas universitarios de mayores.. Universidad de Sevilla. Recuperado el 20 de noviembre de 2013, de http://www.ondosdigitales.us.es/tesis/tesis/1661/variables-psicosociales-queinciden-en-la-calidad-de-vida-del-alumnado-participante-en-programasuniversitarios-de-mayores

GONZÁLEZ BUENO, M. A. (2009). La evaluación como instrumento de mejora y calidad de los programas universitarios para mayores: programa universitario para mayores Peritia et Doctrina de la ULPGC. Universidad de Las Palmas de Gran Canaria.. Recuperado el 15 de noviembre de 2013, de http://acceda.ulpgc.es/bitstream/10553/4865/2/0621493_00000_0000.pdf

LIRIO CASTRO, J. \& MORALES CALVO, S. (2011). El reto de la formación del profesorado para una enseñanza de calidad en las universidades de mayores. Pedagogía Social. Revista Interuniversitaria, 19, 155-166.

LONDOÑO, C., HERNÁNDEZ, L. M., ALEJO, I. E. \& PULIDO, D. (2013). Diseño y validación de la Escala de Optimismo Disposicional/Pesimismo-EOP. Universitas Psychologica, 12 (1), 139-155.

MARTÍ NOGUERA, J. J.; MARTÍNEZ SALVÁ, F.; MARTÍ VILAR, M. \& MARÍ MOLLÁ, R. (2007) Responsabilidad social universitaria: acción aplicada de valoración del bienestar psicológico en personas adultas mayores institucionalizadas. Recuperado el 2 de diciembre de 2013, de http://polis.revues.org/4161; doi: 10.4000/polis.4161

MELÉNDEZ, J. C., MAYORDOMO, T. \& SALES, A. (2013). Comparación entre ancianos sanos con alta y baja reserva cognitiva y ancianos con deterioro cognitivo. Universitas Psychologica, 12 (1), 73-80.

MORALES MUÑOZ, E. (2011). El profesor-tutor de la UNED Senior. Investigación evaluativa. Madrid: UNED.

PADILLA-GÓNGORA, D., SANTIUSTE BERMEJO, V. \& RUIZ FERNÁNDEZ, M. I. (2013). Empowering Marginalized elders: Proyecto Grundtvig. International Journal of Developmental and Educational Psychology. INFAD. Revista de Psicología, 1(2), 311- 316. 
PÉREZ SERRANO, G. (2011). El conocimiento científico y sus carcomas. Teoría de la Educación. Revista Interuniversitaria, 23 (2), 19-43.

PÉREZ SERRANO, G. (2013). Promoción del Envejecimiento Activo a través de las Universidades de Mayores: UNED Senior. IV Edición Premios Senda. Madrid: Libertad Digital.

PÉREZ SERRANO, M. \& PÉREZ SERRANO, G. (2010) Investigación cualitativa. En De Miguel Badesa, S. \& Cerrillo Martín, R. (Coords.) Formación para la inclusión laboral de personas con discapacidad intelectual, 265-279. Madrid: Ediciones Pirámide.

PETERSON, CH. \& SEligMAN, M. (2004). Character Strengths and Vitues. A Handbook and Classification. New York: Osford University Press.

PUGA, D., ROSERO-BIXBY, L., GLASER, K. \& CASTRO, T. (2007). Red social del adulto mayor en perspectiva comparada: Costa Rica, España e Inglaterra. Población y Salud en Mesoamérica. Revista electrónica. 5 (1). Número especial CRELESCosta Rica: Estudio de Longevidad y Envejecimiento Saludable. Recuperado el 5 de diciembre de 2013, de http://ccp.ucr.ac.cr/revista/

SELIGMAN, M. E. (2011). Flourish: A visionary new understanding of happiness and wellbeing. New York: Free Press.

SITGES MACIÁ, E. \& BONET LÓPEZ, B. (2011). Estado emocional y satisfacción de un programa universitario para mayores. International Journal of Development and Educational Psychology. INFAD. Revista de Psicología, 1 (4), 133-142.

TARRAGONA, M. (2013). Psicología Positiva y Terapias Constructivas: Una propuesta Integradora. Terapia Psicológica, 31 (1), 115-125.

VECINA JIMENEZ, M.L. (2006). Emociones positivas. Papeles de Psicología, 27 (1), 12.

VILAPLANA PRIETO, C. (2010). Relación entre los Programas Universitarios para Mayores, la satisfacción durante la jubilación y la calidad de vida. Revista de Investigación Educativa, 28 (1), 195-216. 


\section{Correspondencia con los autores}

Ana FERNANDEZ-GARCIA

Calle Juan del Rosal, 14.

Ciudad Universitaria - UNED

28040 Madrid

e-mail: anafernandez@bec.uned.es

José Luis GARCÍA LLAMAS

Calle Juan del Rosal, 14.

Ciudad Universitaria - UNED

28040 Madrid

e-mail: jlgarcia@edu.uned.es

Gloria PÉREZ SERRANO

Calle Juan del Rosal, 14.

Ciudad Universitaria - UNED

28040 Madrid

e-mail: gloriaperez@edu.uned.es 